Case Report

\title{
Placenta Accreta following Hysteroscopic Lysis of Adhesions Caused by Asherman's Syndrome: A Case Report and Literature Review
}

\author{
Yuko Sonan ${ }^{(D)},{ }^{1}$ Shigeru Aoki, ${ }^{1}$ Kimiko Enomoto, ${ }^{1}$ Kazuo Seki, ${ }^{1}$ and Etsuko Miyagi ${ }^{2}$ \\ ${ }^{1}$ Perinatal Maternity and Neonatal Center of Yokohama City University Medical Center, Yokohama, Japan \\ ${ }^{2}$ Department of Obstetrics and Gynecology, Yokohama City University Hospital, Yokohama, Japan \\ Correspondence should be addressed to Yuko Sonan; aiu525@yahoo.co.jp
}

Received 21 February 2018; Accepted 6 May 2018; Published 31 May 2018

Academic Editor: Julio Rosa-e-Silva

Copyright (c) 2018 Yuko Sonan et al. This is an open access article distributed under the Creative Commons Attribution License, which permits unrestricted use, distribution, and reproduction in any medium, provided the original work is properly cited.

\begin{abstract}
Asherman's syndrome is defined as partial or complete obstruction of the uterine cavity primarily caused by intrauterine procedures and infections. Hysteroscopic adhesiolysis is commonly used to treat Asherman's syndrome. Although the frequency of placenta accreta is known to increase with pregnancy after hysteroscopic adhesiolysis, precise data remain unknown. We report a case of placenta accreta following hysteroscopic lysis of adhesions caused by Asherman's syndrome and IVF treatment and review the literature on placenta accreta following hysteroscopic adhesiolysis. It is necessary to consider placenta accreta as a complication of pregnancies after hysteroscopic adhesiolysis for Asherman's syndrome, particularly in those conceived using IVF.
\end{abstract}

\section{Introduction}

Asherman's syndrome is defined as partial or complete obstruction of the uterine cavity due to damage to the basal layer of the endometrium $[1,2]$ and is primarily caused by intrauterine procedures and infections often associated with miscarriage or curettage for postpartum placental retention [1].

Hysteroscopic adhesiolysis is commonly used to treat Asherman's syndrome. Although the frequency of placenta accreta is known to increase with pregnancy after hysteroscopic adhesiolysis, precise data remain unknown.

Here, we report a case of placenta accreta following hysteroscopic lysis of adhesions caused by Asherman's syndrome and IVF treatment and review the literature on placenta accreta following hysteroscopic adhesiolysis.

\section{Case Presentation}

The patient was a 40-year-old primiparous woman. She was diagnosed with submucosal fibroids by her previous gynecologist 5 years prior, based on chief complaints of atypical genital bleeding and hypermenorrhea. She underwent hysteroscopic myomectomy for one $1 \mathrm{~cm}$ sized and one $3 \mathrm{~cm}$ sized submucosal fibroid located between 2 and 3 o'clock in the uterine fundus. Asherman's syndrome was suspected after the patient exhibited secondary hypomenorrhea 10 months after surgery. Therefore, hysterosalpingography and magnetic resonance imaging (MRI) were performed. Intrauterine adhesions were suspected based on hysterosalpingography findings, while uterine cavity narrowing was identified using MRI. Hysteroscopy revealed filmy adhesions consistent with myomatous tissue at the excision site, and the patient was diagnosed with Asherman's syndrome.

Eight months after diagnosis, the patient underwent hysteroscopic adhesiolysis. The filmy adhesions observed on the left side of the fundus were easily separated with Hegar cervical dilators, and an intrauterine device was inserted after dilation. The patient was diagnosed with stage I Asherman's syndrome defined by European Society for Hysteroscopy classification of intrauterine adhesions, and menstrual flow returned to normal after the operation.

While the patient had a strong desire to bear children, her inability to conceive for 7 years led her to pursue in vitro fertilization (IVF). After having a miscarriage at 7 weeks 


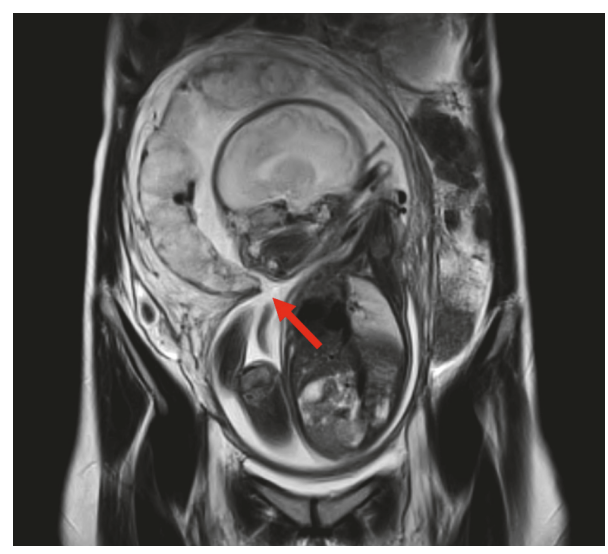

FIgURE 1: Semicircular stenosis, thought to influence adhesions in the uterus, was confirmed, and amniotic membrane sheets (tissues with a free edge visualized within the amniotic cavity) were identified (arrow).

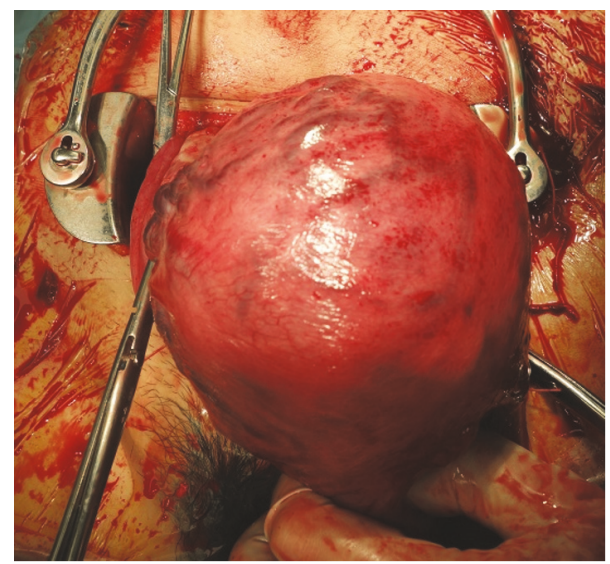

FIGURE 2: Intraoperative finding: blood vessels on the uterine surface at the placental implantation site are engorged.

of gestation, she underwent cervical dilatation and uterine curettage.

Six months after the miscarriage, the patient became pregnant again through IVF and was referred to our hospital at 7 weeks of gestation. At 19 weeks of gestation, tissues with a free edge were visualized within the amniotic cavity using obstetric ultrasound and were determined to be amniotic sheets on MRI at 31 weeks of gestation (Figure 1). The course of pregnancy was uneventful thereafter, and an elective cesarean section was performed at 38 weeks and 2 days of gestation because of a breech presentation.

The placenta adhered to the uterine wall after childbirth and could not be easily separated manually. The blood vessels on the uterine surface at the placental implantation site were engorged (Figure 2), leading us to diagnose the patient with placenta increta. The placenta remained firmly adherent to the uterine wall, and although there was almost no bleeding from the uterine cavity, cesarean hysterectomy was performed after informed consent was obtained from the patient. In the abdominal cavity, $4 \mathrm{~cm}$ subserosal uterine

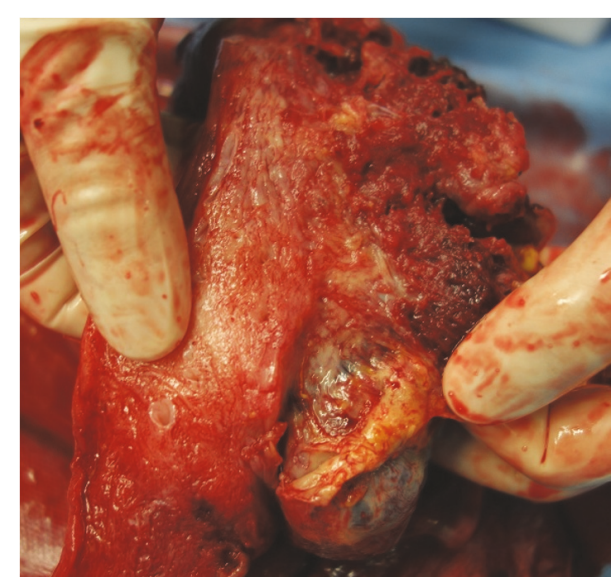

FIGURE 3: Resected uterus: placenta is present from the fundus to the posterior uterine wall. The placenta adheres diffusely to the myometrium, and partial thinning is visible at the fundus.

fibroids were observed on the left side of the fundus, and adhesions thought to be caused by endometriosis were found in the right adnexa, posterior uterus, and anterior rectum. The operative time was 101 minutes, while the total blood loss was $1,584 \mathrm{ml}$ (including amniotic fluid). Blood transfusion was not required. Macroscopic examination of the uterus after extraction showed the presence of placenta from the fundus to the posterior wall, diffusely adherent to the myometrium (Figure 3), along with partial thinning of the fundus.

Placenta increta was confirmed based on postpartum histological findings of placental villi invading the myometrium, without an interposed decidual plate.

The postoperative course was uneventful, and the patient was discharged in good health on the 7th postpartum day.

\section{Discussion}

This case highlights two points: placenta accreta must be considered as a potential complication in pregnancies after hysteroscopic adhesiolysis for Asherman's syndrome, and IVF may further increase the risk of placenta accreta.

First, we should be aware of the possibility of placental implantation disorders, placenta accrete in particular in pregnancies following hysteroscopic adhesiolysis. This patient underwent hysteroscopic adhesiolysis for intrauterine adhesions. Although approximately $90 \%$ of intrauterine adhesions are associated with intrauterine curettage in pregnant women, they can also occur in a nongravid uterus as a result of procedures like myomectomy and curettage that damage the endometrium [13]. Intrauterine adhesions are among the main long-term complications associated with hysteroscopic myomectomy [14], and Al-Inany [13] reported that multiple myomectomies are more likely to cause intrauterine adhesions than a single myomectomy. The amniotic sheets observed in this case may have been related to the intrauterine adhesions resulting from hysteroscopic myomectomy and the $\mathrm{D} \& \mathrm{C}$ performed because of a miscarriage. 
TABLE 1: Pregnancies after hysteroscopic adhesiolysis.

\begin{tabular}{|c|c|c|c|c|}
\hline Study & $\begin{array}{c}\text { Delivery following } \\
\text { hysteroscopic adhesiolysis, } \\
n\end{array}$ & $\begin{array}{c}\text { Adherent placenta, } n \\
(\%)\end{array}$ & $\begin{array}{c}\text { Placenta accreta, } n \\
(\%)\end{array}$ & $\begin{array}{c}\text { Postpartum } \\
\text { hemorrhage, } n(\%)\end{array}$ \\
\hline Chen et al. (2017) [1] & 140 & $6(4.3)$ & $3(2.1)$ & $11(7.9)$ \\
\hline Fernandez et al. (2006) [2] & 21 & 0 & $3(14.3)$ & $3(14.3)$ \\
\hline Roy et al. (2010) [3] & 32 & $3(9.4)$ & $1(3.1)$ & $5(12.5)$ \\
\hline Yu et al. (2008) [4] & 25 & $3(12.0)$ & $2(8.0)$ & - \\
\hline Zikopoulos et al. (2004) [5] & 20 & 0 & $2(10.0)$ & - \\
\hline Capella-Allouc et al. (1999) [6] & 9 & 0 & $2(22.2)$ & - \\
\hline Bhandari et al. (2015) [7] & 10 & $1(10.0)$ & 0 & $1(10.0)$ \\
\hline Liu et al. (2014) [8] & 49 & $1(2.0)$ & $2(4.1)$ & $2(4.1)$ \\
\hline Katz et al. (1996) [9] & 59 & 0 & 0 & 0 \\
\hline Friedman et al. (1986) [10] & 23 & $1(8.7)$ & $1(8.7)$ & - \\
\hline Valle and Sciarra (1988) [11] & 114 & - & $1(0.88)$ & - \\
\hline Feng et al. (1999) [12] & 145 & $1(0.7)$ & 0 & - \\
\hline Total & 647 & $16(2.5)$ & $17(2.6)$ & $22(3.4)$ \\
\hline
\end{tabular}

Table 1 shows the results of a literature review performed by searching MEDLINE for pregnancy outcomes following hysteroscopic adhesiolysis for Asherman's syndrome [1-12]. Although the reported frequency of subsequent placenta accreta varies, a review of 12 studies published between 1986 and 2016 showed that 647 of such pregnancies ended in live birth. Of these, 17 women were diagnosed with placenta accreta, at a rate of $2.6 \%$ (17/647). Additionally, many, but not all, cases described adherent placenta and retained placenta without concomitant placenta accreta. Xiao et al. [15] reported that 64/201 cases (31.8\%) had retained placenta, adherent placenta, or placenta accreta, and postpartum hemorrhage (PPH) occurred in 127/221 (63.2\%) cases, suggesting that not only placenta accreta but also PPH must be considered in pregnancies after hysteroscopic adhesiolysis.

Second, IVF may increase the risk of placenta accreta.

The incidence of placenta accreta is significantly higher in IVF pregnancies than in spontaneous pregnancies [16]. EshBroder et al. [16] reported that the rate of placenta accreta in spontaneous pregnancies was $12 / 752(1.2 / 1000)$, while that in IVF pregnancies was $30 / 24,441(16 / 1,000)$. The higher rate of placenta accreta in IVF pregnancies may be due to the change in endometrial environment and morphological and structural changes to the endometrium due to the IVF treatment protocol (stimulation protocol) [16].

Intrauterine adhesions (IUA) severity is associated with greater reduction in fertility $[1,3,17]$, thereby increasing the likelihood that affected patients will need to undergo IVF to have a successful pregnancy. The extent of endometrial loss increases with IUA severity, which likely confers a greater risk of placenta accreta. For this reason, doctors should be aware that IVF pregnancy following hysteroscopic adhesiolysis involves a greater risk of placenta accreta.

\section{Conclusion}

Placenta accreta should be considered as a complication of pregnancies after hysteroscopic adhesiolysis for Asherman's syndrome, particularly in those conceived using IVF.

\section{Conflicts of Interest}

The authors declare that they have no conflicts of interest.

\section{Authors' Contributions}

Yuko Sonan and Shigeru Aoki contributed to the study design and finalization of the manuscript. Kimiko Enomoto and Kazuo Seki wrote the first draft of the manuscript. Etsuko Miyagi provided the study design and supervised the study.

\section{References}

[1] L. Chen, H. Zhang, Q. Wang et al., "Reproductive Outcomes in Patients With Intrauterine Adhesions Following Hysteroscopic Adhesiolysis: Experience From the Largest Women's Hospital in China," Journal of Minimally Invasive Gynecology, vol. 24, no. 2, pp. 299-304, 2017.

[2] H. Fernandez, F. Al-Najjar, A. Chauveaud-Lambling, R. Frydman, and A. Gervaise, "Fertility after treatment of Asherman's syndrome stage 3 and 4," Journal of Minimally Invasive Gynecology, vol. 13, no. 5, pp. 398-402, 2006.

[3] K. K. Roy, J. Baruah, J. B. Sharma, S. Kumar, G. Kachawa, and N. Singh, "Reproductive outcome following hysteroscopic adhesiolysis in patients with infertility due to Asherman's syndrome," Archives of Gynecology and Obstetrics, vol. 281, no. 2, pp. 355-361, 2010.

[4] D. Yu, T. C. Li, E. Xia, X. Huang, Y. Liu, and X. Peng, "Factors affecting reproductive outcome of hysteroscopic adhesiolysis 
for Asherman's syndrome," Fertility and Sterility, vol. 89, no. 3, pp. 715-722, 2008.

[5] K. A. Zikopoulos, E. M. Kolibianakis, P. Platteau et al., "Live delivery rates in subfertile women with Asherman's syndrome after hysteroscopic adhesiolysis using the resectoscope or the Versapoint system," Reproductive BioMedicine Online, vol. 8, no. 6, pp. 720-725, 2004.

[6] S. Capella-Allouc, F. Morsad, C. Rongières-Bertrand, S. Taylor, and H. Fernandez, "Hysteroscopic treatment of severe Asherman's syndrome and subsequent fertility," Human Reproduction, vol. 14, no. 5, pp. 1230-1233, 1999.

[7] S. Bhandari, P. Bhave, I. Ganguly, A. Baxi, and P. Agarwal, "Reproductive outcome of patients with Asherman's syndrome: A SAIMS experience," Journal of Reproduction and Infertility, vol. 16, no. 4, pp. 229-235, 2015.

[8] X. Liu, H. Duan, and Y. Wang, "Clinical characteristics and reproductive outcome following hysteroscopic adhesiolysis of patients with intrauterine adhesion-a retrospective study," Clinical and Experimental Obstetrics and Gynecology, vol. 41, pp. 144-148, 2014.

[9] Z. Katz, A. Ben-Arie, S. Lurie, M. Manor, and V. Insler, "Reproductive outcome following hysteroscopic adhesiolysis in Asherman's syndrome," International Journal of Fertility and Menopausal Studies, vol. 41, no. 5, pp. 462-465, 1996.

[10] A. Friedman, J. Defazio, and A. Decherney, "Severe obstetric complications after aggressive treatment of asherman syndrome," Obstetrics \& Gynecology, vol. 67, no. 6, pp. 864-867, 1986.

[11] R. F. Valle and J. J. Sciarra, "Intrauterine adhesions: hysteroscopic diagnosis, classification, treatment, and reproductive outcome," American Journal of Obstetrics \& Gynecology, vol. 158, no. 6I, pp. 1459-1470, 1988.

[12] Z. C. Feng, B. Yang, J. Shao, and S. Liu, "Diagnostic and therapeutic hysteroscopy for traumatic intrauterine adhesions after induced abortions: clinical analysis of 365 cases," Gynaecological Endoscopy, vol. 8, no. 2, pp. 95-98, 1999.

[13] H. Al-Inany, "Intrauterine adhesions: an update," Acta Obstetricia et Gynecologica Scandinavica, vol. 80, no. 11, pp. 986-993, 2001.

[14] O. Taskin, S. Sadik, A. Onoglu et al., "Role of endometrial suppression on the frequency of intrauterine adhesions after resectoscopic surgery," The Journal of Minimally Invasive Gynecology, vol. 7, no. 3, pp. 351-354, 2000.

[15] S. Xiao, Y. Wan, M. Xue et al., "Etiology, treatment, and reproductive prognosis of women with moderate-to-severe intrauterine adhesions," International Journal of Gynecology and Obstetrics, vol. 125, no. 2, pp. 121-124, 2014.

[16] E. Esh-Broder, I. Ariel, N. Abas-Bashir, Y. Bdolah, and D. H. Celnikier, "Placenta accreta is associated with IVF pregnancies: A retrospective chart review," BJOG: An International Journal of Obstetrics \& Gynaecology, vol. 118, no. 9, pp. 1084-1089, 2011.

[17] J. Zhao, Q. Chen, D. Cai, Z. Duan, X. Li, and X. Xue, "Dominant factors affecting reproductive outcomes of fertilitydesiring young women with intrauterine adhesions," Archives of Gynecology and Obstetrics, vol. 295, no. 4, pp. 923-927, 2017. 


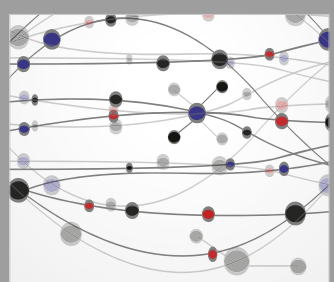

The Scientific World Journal
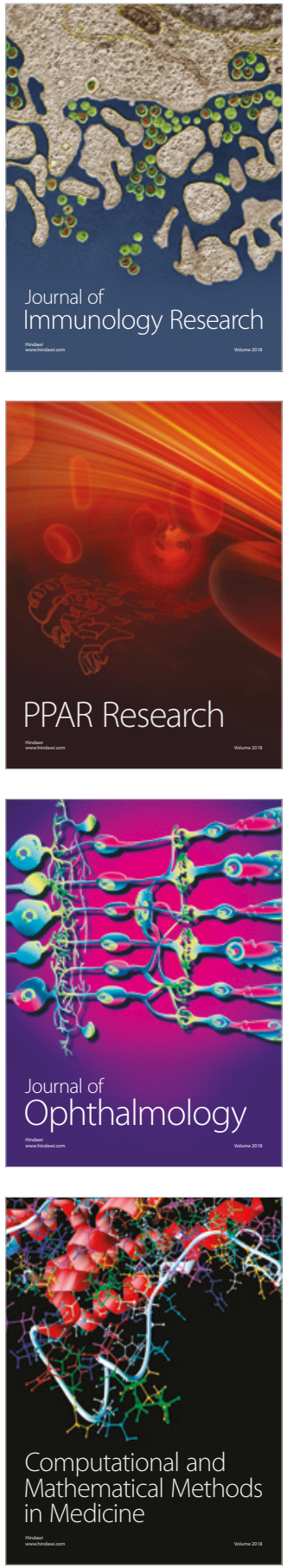

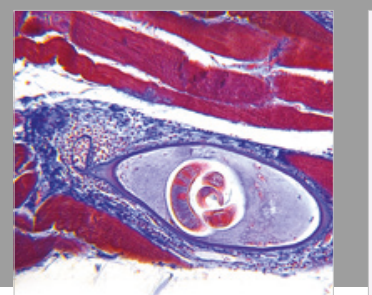

Gastroenterology Research and Practice

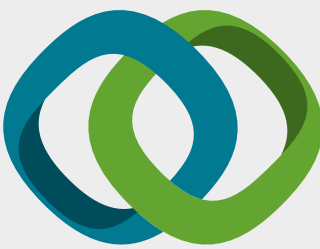

\section{Hindawi}

Submit your manuscripts at

www.hindawi.com
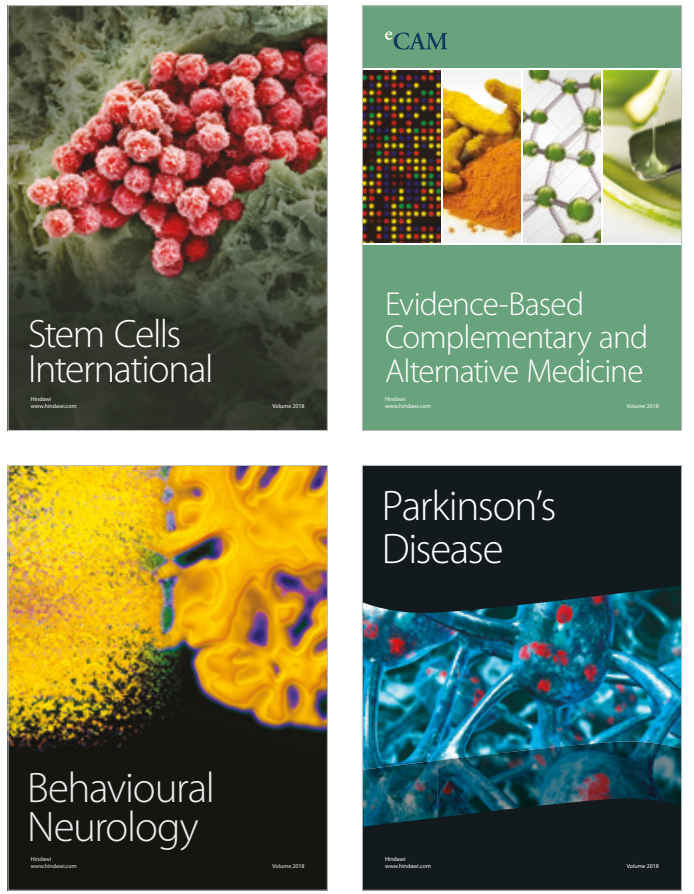

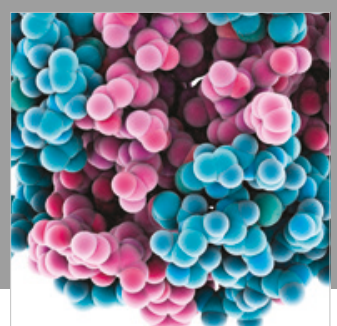

ournal of

Diabetes Research

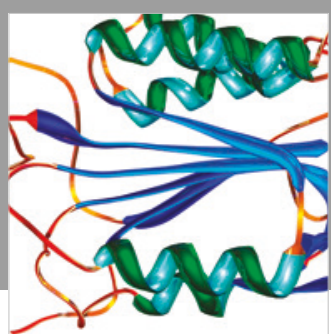

Disease Markers
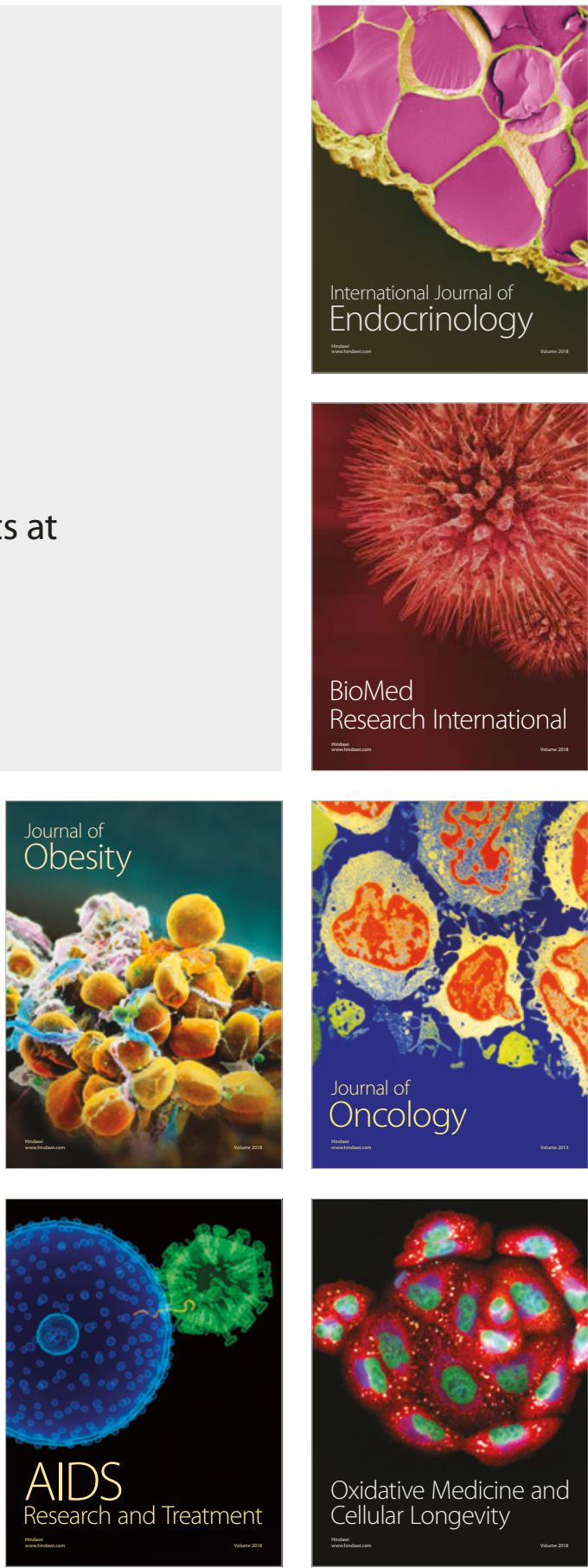